\title{
La relación entre los centros escolares, las familias y los entornos comunitarios como factor de calidad de la educación de menores y adultos (Presentación del monográfico)
}

\author{
FranCISCO-JUAN GARCía-BACETE \\ Y RAQUEL-AMAYA MARTÍNEZ-GONZÁLEZ \\ Universitat Jaume I, Castellón; Universidad de Oviedo
}

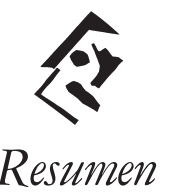

Este artículo destaca la necesidad de fomentar las relaciones de cooperación entre los centros escolares, las familias y los entornos sociales comunitarios por actuar todos ellos como contextos y agentes educativos y de socialización que, por separado no pueden llegar a responder de manera eficaz a las necesidades de desarrollo y formación de menores y adultos. A este respecto, se describen e introducen en este artículo los trabajos que configuran este número monográfico, que tratan de conceptualizar el valor social de esta cooperación, de ofrecer resultados de investigación que muestran la necesidad de llevarla a efecto, y de aportar propuestas metodológicas y experienciales en centros y en entornos comunitarios que permitan hacerla realidad.

Palabras clave: Cooperación entre centros escolares, familias y entornos comunitarios, agentes educativos y de socialización, menores, adultos.

\section{The relationship between school, family and community setting as quality factor in adult and children's education (Introduction to monograph)}

\begin{abstract}
The need to promote effective school-family-community partnership is emphasized given that these three social environments act as educational and socialization agents that separately cannot effectively respond to the developmental and educational needs of children and adults. The papers that make up this monographic issue are introduced and described. Their authors attempt to conceptualize the social value of school-family-community partnership, to offer research results that demonstrate the need for this partnership, and to contribute metbodologies and experiences for the school and community settings so that they may be put into practice.

Keywords: Effective school-family-community partnership, educational and socialization agents,
\end{abstract} children, adults. 


\section{Cultura y Educación, 2006, 18 (3-4), pp. 213-218}

Desde perspectivas comunitarias y ecológicas (Bronfenbrenner, 1979, 1986) se entiende que escuelas y familias son contextos educativos y de desarrollo no sólo de niños y jóvenes sino también de adultos, ya sean profesores y profesoras, madres y padres u otras personas con responsabilidades educativas y familiares. Los centros escolares reúnen características que los convierten en escenarios privilegiados de socialización de los niños y adolescentes, y también de los adultos. El profesorado ve facilitada su integración en el centro y en el barrio donde éste se ubica a través de los compañeros, de los padres y madres de sus alumnos y de otros agentes sociales comunitarios con quienes comparte responsabilidades y actividades educativas. Por su parte, los padres y madres se socializan con otros padres y madres de los compañeros de sus hijos, compartiendo experiencias y proyectos que no están sólo centrados en la educación de los hijos, sino también en su propia formación como padres y madres, en la mejora del funcionamiento e instalaciones de los centros escolares, en la promoción de las tradiciones y en la generación de nuevos recursos para la comunidad. Los adultos, profesorado, madres y padres, agentes sociales y comunitarios, pueden, y deben, aprovechar este escenario educativo para construir redes sociales de apoyo mutuo, tanto para sí como para sus hijos de forma sistemática y organizada a través, por ejemplo, de las Asociaciones de Padres y Madres de Alumnos (AMPAs), de asociaciones vecinales o de otras formas de asociación.

Es claro que los centros académicos no pueden por sí solos proporcionar a los menores todos los aprendizajes necesarios para que puedan desarrollarse íntegramente y adaptarse adecuadamente a la sociedad, por eso se enfatiza el papel de los padres y madres y de otros adultos con responsabilidades familiares en la educación de los niño/as, y la necesidad de que se establezcan vínculos estrechos de cooperación entre las familias, los centros docentes y las entidades sociales comunitarias. Las relaciones sistemáticas y de colaboración entre la escuela y las familias contribuyen a asegurar la necesaria continuidad entre la educación adquirida en el hogar y la que se obtiene en los centros escolares, y a que estos dos agentes de socialización potencien mutuamente sus roles educativos en sus respectivos contextos de desarrollo y de aprendizaje. La institución educativa ha de incorporar entre sus objetivos el continuo y progresivo apoyo a la acción educadora de las familias y éstas, a su vez, han de participar en el diseño y desarrollo de los proyectos educativos de los centros escolares. Se han de crear marcos de relación que hagan posible incorporar prácticas diversas desde niveles de actuación también diversos: a nivel institucional, a nivel de cada centro y de cada AMPA, a nivel de aula y profesor individual y de familia concreta. Esto se hace especialmente necesario en el periodo de la educación obligatoria de los niños y jóvenes.

No obstante, la realidad actual dista bastante de parecerse al escenario descrito, no tanto por falta de capacidad potencial, como por la falta de tradición, de formación, de confianza y de asunción de nuevos papeles de unos y otros, de pérdida del valor de lo educativo en los centros y en las familias, desbordados por otras demandas y otros intereses.

No se trata tanto de realizar más o menos actividades de colaboración entre padres y profesorado, como de ir avanzando en cada una de ellas superando las dificultades que se encuentren, de atreverse a innovar, a experimentar la alianza y de realizar el esfuerzo de orientarlas, liderarlas y sistematizarlas. La dinamización de las relaciones escuela-familia se va desarrollando progresivamente a través de proyectos de innovación en los centros, pero dado que la energía del profesorado no es inagotable, se ha de realizar un concienzudo examen de en qué se invierte esta energía. Muchos expertos han propuesto el término "partenariado" ("partnership”, Allexsaht-Snider, Martínez-González, y Phtiaka, 1996; Epstein, 1997; Macbeth, y Ravn, 1994; Martínez-González, Rodríguez-Ruíz, Pérez-Herrero y 
Torío-López, 2005) para referirse al proceso que conlleva que padres y profesorado aprendan a trabajar juntos, valorando lo que cada parte puede aportar a esta relación. Y es dentro de este marco donde se ubica la aportación de este número monográfico.

En otros trabajos los coordinadores de este Monográfico han presentado algunos factores que pueden hacer más eficaces los esfuerzos de colaboración entre familias y escuelas (García-Bacete, 2003; Martínez-González et al., 2000). En primer lugar, parece requisito indispensable entender que la colaboración escuela-familia constituye una respuesta necesaria a las actuales necesidades sociales; desde esta perspectiva la escuela adquiere una dimensión de servicio a las necesidades del alumnado y de sus familias, y éstas, por su parte, han de contribuir al desarrollo de los niños y a su éxito académico. Mejorar la comunicación entre ambos agentes educativos y reflexionar sobre los instrumentos de intercambio de información entre padres y profesorado ha de ser un objetivo prioritario en la construcción de un camino hacia relaciones caracterizadas por la "reciprocidad" (igualdad de estatus) y la "mutualidad" (tener asuntos en común) (Forest y García-Bacete, 2006; Vanderlisce y García-Bacete, 2006).

En segundo lugar, es preciso considerar la diversidad de las familias y de las formas de implicación de los padres en la educación de sus hijos y en los centros escolares, lo que conlleva un doble requerimiento: a) Incrementar el conocimiento sobre las principales dimensiones en las que las familias pueden variar e influir en la educación de sus hijos, y sobre las creencias, los valores y estrategias educativas de los padres, y b) Aceptar que existen diversas formas de implicación de los padres, todas potencialmente válidas si son percibidas como factibles por padres y profesores y articuladas de la manera adecuada.

En tercer lugar, es importante valorar los aspectos organizativos de centro que facilitan la implicación de los padres, la explicitación de su participación en el proyecto educativo de centro, y considerar que la iniciativa de fomentar dicha implicación debe partir de la escuela con el apoyo de la dirección del centro y de la Administración educativa a través de la normativa existente al respecto y de la financiación necesaria.

En cuarto lugar, es preciso también considerar el valor de lo particular y recordar que los padres se interesan especialmente por aquello que está directamente vinculado con sus propios hijos, y que su implicación en las actividades del centro se incrementa cuando las prácticas escolares responden a sus necesidades particulares.

En quinto lugar, es necesario también hacer constar que la formación inicial y continua del profesorado requiere incorporar en sus programas temas, estrategias y metodologías de investigación-acción que le faciliten desarrollar en los centros iniciativas que promuevan una eficaz cooperación con las familias.

Por último, es preciso considerar que los entornos comunitarios en los que se desenvuelven los menores, los centros escolares y las familias ejercen también una influencia educativa importante en el desarrollo de niños y adultos, por lo que conviene estudiar la colaboración que cabe establecer entre las familias, los centros docentes y las entidades y servicios sociales comunitarios tanto desde un punto de vista teórico como aplicado, como se ha hecho constar en la $5^{\text {a Confe- }}$ rencia Internacional de la Red Europea de Investigación en Educación Familiar (ERNAPE) (Family-School-Community Partnerships) celebrada en Oviedo (Martínez-González et al., 2005).

Partiendo de la idea de que reciprocidad y mutualidad son dos características que han de estar presentes en las relaciones que se establezcan entre el profesorado y los padres, en este monográfico se trata de analizar como piensan y como viven los profesores las relaciones con los padres y sus necesidades de formación 
al respecto, dado que parece necesario que sean los centros escolares y el profesorado quienes tomen la iniciativa de propiciar y mantener estas relaciones. Por ello, en este Monográfico se analiza también la posibilidad de crear grupos de trabajo en los centros que actúen como dinamizadores de las relaciones, y que se promuevan desde los centros y la comunidad redes de apoyo que faciliten la continuidad educativa entre el contexto familiar y escolar.

En la selección de artículos incluidos en este monográfico se ha tenido en cuenta tanto la complementariedad de temas y enfoques para asegurar una perspectiva multidisciplinar, como la diversidad de instituciones representadas y su ubicación geográfica nacional e internacional para alcanzar una mayor representatividad y significatividad en las temáticas tratadas.

Los artículos que se incluyen pretenden contribuir a conocer la realidad actual de las relaciones que se establecen entre los centros educativos, las familias y la comunidad y a realizar propuestas de cambio que faciliten promover y mejorar dichas relaciones. Cada artículo ofrece aportaciones específicas al tema, que permiten completar y complementar distintos ángulos desde los que puede estudiarse esta relación compleja: contribución de los conceptos "capital cultural" y "capital social" a la comprensión de cómo la colaboración entre la escuela y la familia influye en el desarrollo personal de los niños y en su éxito académico; percepción y valoración tanto de los profesores como de los padres de su relaciones mutuas; metodología activa para investigar y promover al mismo tiempo la colaboración entre profesores y padres, y entrenamiento del profesorado para comunicarse eficazmente con los padres; la ampliación del partenariado a los agentes sociales y a la comunidad en general.

En el primer artículo, de carácter conceptual, titulado "Cultural and social capital: can we learn for investigating and strengthening school-family collaboration?" y escrito por el profesor Loizos Symeous del Cyprus Pedagogical Institute, se presentan conceptos teóricos fundamentales que ayudan a entender como las escuelas y las familias influyen en la educación y desarrollo de los niños: el concepto de "capital cultural" desarrollado por P. Bourdieu y el de "capital social" acuñado por J. Coleman, muestran la importancia de la cooperación activa entre escuelas y familias.

En el segundo artículo, de carácter metodológico, titulado "Propuestas metodológicas para una educación de calidad a través de las relaciones entre centros docentes, familias y entidades comunitarias", de las profesoras Raquel-Amaya Martínez-González y $\mathrm{M}^{\mathrm{a}}$ Henar Pérez-Herrero del Departamento de Ciencias de la Educación de la Universidad de Oviedo, se aportan estrategias metodológicas basadas en la investigación-acción para poder dar respuesta al reto de la colaboración entre las familias, los centros educativos y el entorno comunitario. En opinión de las autoras, la investigación-acción, con sus elementos y fases, permite a padres y profesorado colaborar eficazmente para identificar las necesidades de cooperación que existen en los centros y para promover respuestas a las mismas que estén contextualizadas y sean innovadoras. El artículo hace especial hincapié en la consideración de esta metodología en los procesos de formación del profesorado.

El tercer artículo, titulado "Cómo son y cómo podrían ser las relaciones entre escuelas y familias en opinión del profesorado", del profesor Francisco-Juan García-Bacete del Departamento de Psicología Evolutiva y de la Educación de la Universidad Jaume I de Castellón, está basado en la investigación empírica y plantea la necesidad de analizar desde la perspectiva de la ecología social el punto de vista adoptado por los docentes sobre las relaciones entre escuelas y familias, dado su papel esencial de iniciadores y mantenedores de dichas relaciones. En el artículo se abordan cuatro áreas específicas: a) el papel de los padres en la escolarización de sus hijos; b) cómo son las relaciones entre las familias y las escuelas y viceversa; c) 
cómo piensan que deberían ser estas relaciones, si fuera el caso; y d) cuáles son las principales barreras que dificultan el establecimiento de relaciones sistemáticas entre profesores y padres.

El cuarto artículo, también empírico, titulado "What parents and teachers think about their schools and each other" de Sam Redding, Director del Academic Development Institute de Illinois (USA), define el concepto de "comunidad educativa" y analiza las percepciones que tienen padres y profesorado de centros americanos de enseñanza primaria y secundaria ubicados tanto en ámbitos urbanos como rurales con estatus socio-económico muy bajo, acerca del centro escolar y de las relaciones que mantienen entre ellos. Se estudian también las opiniones de los padres sobre la influencia que tienen los procesos familiares en los resultados académicos de los hijos

El quinto artículo describe una experiencia educativa dirigida a "Romper las barreras entre la familia y la escuela", escrita por el profesor Enrique-Javier Díez Gutiérrez del Departamento de Didáctica y Organización Escolar de la Universidad de León y Eloína Terrón Bañuelos, Trabajadora Social del Equipo de Orientación Educativa y Psicopedagógica de Valencia de D. Juan (León) de la Junta de Castilla-León. En el artículo se expone un programa de formación y asesoramiento en habilidades de comunicación dirigido al profesorado y a las familias con el objetivo de romper las tradicionales barreras de relación que existen entre ambos agentes educativos. Su aportación más innovadora es poner la tecnología al servicio de la formación; en concreto, el programa se articula en torno a un vídeo diseñado y elaborado expresamente para el programa, en el que se puede observar distintos estilos de entrevistas entre un profesor y una madre.

El sexto artículo, "Nuevas alternativas y modelos en la relación familia-escuela" describe también una experiencia con la que su autora, la profesora Maria Jesús Comellas Carbó, del Departamento de Pedagogía Aplicada de la Universidad Autónoma de Barcelona, fomenta la idea de que los entornos comunitarios tienen muchas potencialidades educativas. Mediante un modelo de investigación-acción liderado por ayuntamientos, se crea una red multiprofesional implicada en las actividades del municipio, que junto con representantes de las Asociaciones de Padres y Madres de Alumnos (AMPAs) debaten diferentes enfoques educativos que emergen de cada colectivo para crear una línea educativa coherente y compartida que favorezca la formación de los menores y también de los adultos.

En el último trabajo "Guía de Recursos para promover las relaciones entre escuelas y familias", elaborado por el profesor Francisco-Juan García-Bacete, se inventarían y comentan algunos de los principales recursos (bibliográficos, enlaces, revistas, organizaciones) disponibles en nuestro contexto para promover la colaboración entre los centros escolares, las familias y las entidades comunitarias como factor de calidad en la educación de menores y adultos.

\section{Referencias}

Allexsaht-Snider, M., Martínez GonzÁlez, R. A. \& Phtiaka, H. (1996). International perspectives: Preparing teachers for partnership. Documento presentado a la Education is Partnership Conference. Copenhague, Noviembre. Organizado por la European Research Network About Parents in Education (ERNAPE).

Bronfenbrenner, U. (1979). The ecology of human development. Cambridge: Harvard University Press.

Bronfenbrenner, U. (1986). Ecology of the family as a context for human development: Research perspectives. Developmental Psychology, 22 (6), 723-742.

EPSTEIN, J. (1997). School, family and community partnership. Thousand Oaks, CA: Corwin Press, INC.

FOREST, C. \& GARCÍA-BACETE, F. J. (2006). Comunicación cooperativa entre la familia y la escuela. Valencia: Nau LLibres.

GARCÍA-BACETE, F. J. (2003). Las relaciones escuela-familia: un reto educativo. Infancia y Aprendizaje, 26 (4), $425-437$.

Macbeth, A. \& RAVN, B. (Eds.) (1994). Expectations about parents in education. European Perspectives. Glasgow: European Parents' Association. 
218 Cultura y Educación, 2006, 18 (3-4), pp. 213-218

Martínez González, R. A., Pereira González, M., Rodríguez Díez, B., Peña del Agua, A., Martínez Álvarez, R., GarCía González, M. P., Donaire Rubio, B., Álvarez, A. I. \& Casielles Muñoz, V. (2000). Dinamización de las relaciones familia-Centro escolar a través de la formación del profesorado en este campo de actuación. Revista Española de Orientación y Psicopedagogía, 11 (19), 107-120.

Martínez-González R. A., Rodríguez-Ruíz, B., Pérez-Herrero M. H. \& Torío-López, S. (2005, Eds.). Family-School-Community Partnerships Merging into Social Development. Oviedo: Grupo SM.

VANDERLISCE, V. J. \& GARCÍA-BACETE, F. J. (2006). Comunicación para la potenciación. Un manual de técnicas de enseñanza potenciadoras para facilitadores. Valencia: Nau Llibres. 